\title{
EFFECTS OF HUMAN PLATELET LYSATES WITHOUT ADDITIONAL GROWTH FACTORS ON THE PROTEIN PROFILES OF HUMAN UMBILICAL VEIN ENDOTHELIAL CELL CULTURE MEDIA
}

\author{
HAZRIANI RA, LISA AMIR, RATNA FARIDA*
}

Department of Oral Biology, Faculty of Dentistry, Universitas Indonesia, Depok, Indonesia. Email: friedakuayu@yahoo.co.id Received: 21 April 2017, Revised and Accepted: 13 July 2017

\section{ABSTRACT}

Objectives: The objective of this study is to evaluate the effect of HPLs with no additional GFs on the HUVEC protein profile.

Methods: HUVEC cultures were examined in groups as follows: Fetal bovine serum (FBS), 2\%-HPL with a GF, and 2\%- and 5\%-HPL without a GF which were analyzed with a sodium dodecyl sulfate-polyacrylamide gel electrophoresis test.

Results: The intensity, thickness, and molecular weight of HUVEC band proteins cultured without a GF were not significantly different compared to the control groups (FBS or HPL with a GF).

Conclusions: No difference was found in the HUVEC protein profile after they were cultured with FBS and HPLs, with or without GFs.

Keywords: Fetal bovine serum, Growth factor, Human platelet lysate, Human umbilical vein endothelial cell, Protein profile.

(C) 2017 The Authors. Published by Innovare AcademicSciences PvtLtd. Thisis an open access article underthe CC BY license (http://creativecommons. org/licenses/by/4.0/) DOI: http://dx.doi.org/10.22159/ajpcr.2017.v10s5.23095

\section{INTRODUCTION}

The success of a cell culture depends on the growth medium that is used. Selecting a cell culture medium is related to the cells' environmental requirements in terms of $\mathrm{pH}$ level, temperature, gas concentration, osmolarity, nutrients, and other important factors. Culture media contain amino acids, vitamins, minerals, and glucose, and they have a stable $\mathrm{pH}$ (7.0-7.4), accurate osmolarity, and gases such as $\mathrm{O}_{2}$ and $\mathrm{CO}_{2}$. Cell culture media generally incorporate serums that contain lipids, hormones, and growth factors (GF) [1].

Serum is needed in cell culture medium to protect the cells from damage during treatment and to improve cell adherence [1]. Serum can be obtained from various sources, such as horses, humans, and cows (i.e.,, fetal bovine serum [FBS]). FBS, which is produced from 3-month-old fetal calves, is the most commonly used type of serum. This serum is obtained by inserting a needle into the fetal cow's heart and then draining the blood into a tube using a vacuum. The collected blood is then allowed to clot, and the serum can be obtained after centrifugation [2].

The use of serum FBS in cell culture medium has many disadvantages, including the risk of bacterial contamination, the limited availability of FBS resources, and the cost of production [3]. In an effort to replace the use of FBS in cell culture media, research has been done to find a viable alternative. FBS replacements can be obtained from human bloodderived components, including plasma serum, umbilical cord blood serum, and platelet derivatives such as human platelet lysate (HPL) [4].

HPLs are derived from platelets with a high concentration of blood plasma [5]. HPL contains many GFs that are necessary for cell growth and proliferation. The GFs contained in HPLs include the following: Platelet-derived GF (PDGF), transforming GF (TGF) beta 1, insulinlike GF (IGF-1), basic fibroblast GF (bFGF), epithelial GF (EGF), vascular endothelial GF (VEGF), and hepatocyte GF (HGF) [3]. Using adipose-derived stromal cells (ASC), the results of this study show that ASC HPL cell culture is a suitable alternative to FBS, which may even have better performance [5].
Endothelial cells play an important role in angiogenesis in which new blood vessels are developed from existing blood vessels. Angiogenesis is required for repair, growth, and bone development. This process is influenced by the environment surrounding the cells, and it involves cross-talks between endothelial cells and those of the adjacent bone [6]. Human umbilical vein endothelial cells (HUVECs) are used as an in vitro model of the mechanism of angiogenesis [7].

The most widely used HUVEC culture media are the following: Eagle's minimal essential medium (EMEM), Dulbecco's modified EMEM, Ham's F12 supplement (F12), Roswell Park Memorial Institute Medium 1640, and medium 200. Exogenous GFs, such as VEGF, EGF, and bFGF, need to be added to the culture medium to support the growth and development of HUVECs.

In previous studies, the effectiveness of HPL in HUVEC culture medium has been investigated as a substitute serum [8]. However, the use of HPL in cultured HUVEC supplements to replace GFs is currently unknown. Therefore, this study was conducted to evaluate the effect of the use of HPL without the addition of exogenous GFs on HUVEC culture medium. The analysis was performed on the protein profile of HUVEC culture.

\section{METHODS}

Laboratory research experiments were conducted in vitro using HUVEC culture medium, which had been incubated for 3 days, as a sample. There were four study groups: Two control groups (FBS and 2\%-HPL with a GF) and two treatment groups (2\%-HPL and 5\%-HPL both without a GF). The control group was cultured using medium 200 and an LSGS kit, whereas the treatment groups were cultured using medium 200, 2\%-HPL, 5\%-HPL, and gentamicin.

HPL was obtained by mixing platelets (blood type 0) with plasma (blood type $\mathrm{AB}$ ), and this mixture was centrifuged to obtain platelet rich plasma. Three freeze-Thaw cycles were performed in which the platelet rich plasma was frozen and thawed to lyse the platelets and release the GFs. To obtain the platelet concentrate, all blood samples were centrifuged at high speed to separate into plasma, erythrocytes, and buffy coats. The 
buffy coats were centrifuged again at low speed to obtain a supernatant that contained platelets (i.e., a platelet concentrate).

To determine the protein profile, a sodium dodecyl sulfate-polyacrylamide gel electrophoresis (SDS-PAGE) test was conducted. The total protein concentration in the samples was denatured using a standardized HUVEC culture medium. All the protein concentration data used in this study were based on the Bradford test results. The electrophoresis process was conducted on proteins that had already been denatured. Protein electrophoresis results were then analyzed by measuring the intensity, thickness, and molecular weight of the protein bands.

Protein bands 3 and 4 were analyzed in this study because these bands were always visible in each group. Values of the protein band intensity and thickness were measured using Image J software. Intensity values and measurement data were analyzed by the Shapiro-Wilk normality test, and the obtained data were normally distributed. Then, statistical analysis was performed using one-way analysis of variance (ANOVA; SPSS 18). The statistical analysis had a significance level of 0.05 $(p=0.05)$ and a $95 \%$ confidence level $(\alpha=0.05)$. The molecular weight of the proteins was measured using Gel Doc.

\section{RESULTS}

The average values of intensity of bands 3 and 4 in each group are shown in Fig. 1. The results show that the average intensity level of band 3 in the $2 \%$-HPL group without a GF had the lowest score, whereas that of the 5\%HPL group without a GF was higher than the FBS group and lower than the $\%$-HPL group with GF. For band 4, the average intensity values of the treatment groups (i.e., 2\%-HPL and 5\%-HPL without a GF) were lower than those of the control groups (i.e., FBS and 2\%-HPL with a GF). The mean value of the FBS group had the highest intensity. From the analysis of protein intensity for bands 3 and 4, which was performed with statistical tests (one-way ANOVA), the results showed that there were no significant differences for these bands between the control and treatment groups.

In Table 1, it can be observed that the average thickness of band 3 was higher in one of the treatment groups (i.e., $5 \%$-HPL without a GF) than in the control groups (i.e., FBS and 2\%-HPL with a GF), whereas this average was lower in the $2 \%$-HPL group without a GF. The average thicknesses of band 4 were almost the same as those of band 3 . The $5 \%$-HPL treatment group without a GF had a higher average than the control groups (i.e., FBS and 2\%-HPL with a GF), but the average was higher in the 2\%-HPL group without a GF than that of the FBS group. Overall, the 5\%-HPL group without a GF had the highest thickness value in protein bands 3 and 4 .

\section{DISCUSSION}

The results of this study indicate the protein profiles for the use of HPLs (2\% and 5\%) without GFs in the standard HUVEC culture medium. Quantitative analysis was performed on the SDS-PAGE results for protein bands 3 and 4 . The bands were analyzed by measuring the intensity, thickness, and weight of the protein molecules. The intensity showed on the protein bands indicated the amount of protein in each band, whereas the thickness and weight of the protein molecules showed protein candidates that were expressed by HUVEC after being cultured in the control and treatment medium.

There were no significant differences among the protein profiles for bands 3 and 4 of each HUVEC study group (i.e., those cultured with FBS or HPL with and without GFs). This phenomenon may have been caused by the number of GFs in HPL required for the proliferation and growth of cells. GFs contained within HPLs include PDGF, TGF- $\alpha$, IGF-1, VEGF, fibroblast GF (FGF-2), bFGF, HGF, and EGF, each of which serves as a cell mitogen [4].

Protein profiles were higher in the 5\%-HPL group without a GF than in the other groups. This difference is thought to be caused by the GFs contained in the HPL media such as VEGF, bFGF, and EGF. Based on the previous research on coculture fibroblasts and HUVECs, GFs are capable of stimulating differentiation, mitogenesis, and migration for HUVECs [9]. EGFs also play a role in the proliferation of HUVEC (in vitro) and angiogenesis (in vivo) [9].

The findings in our study showed that the molecular weight of protein bands 3 and 4 did not vary greatly among the groups (Table 2). The lowest molecular weight for protein band 3 was $78.62 \mathrm{kDa}$, whereas the highest molecular weight was $114.22 \mathrm{kDa}$. Based on this data, there are several candidate proteins with a molecular weight that falls outside of band 3, including intercellular adhesion molecule-1 (ICAM-1; CD54), vascular cell adhesion molecule-1 (VCAM-1; CD106), CD34, CD146, and endothelial selectin (E-selectin; CD62E). For protein band 4, the lowest molecular weight was $53.38 \mathrm{kDa}$, whereas the highest molecular weight was $83.83 \mathrm{kDa}$. Protein candidates that may be contained in this band are CD46, CD102 (ICAM-2), and CD93.

ICAM-1 or CD54 is a class of glycoprotein with a 75-115 kDa molecular weight that is found on the cell surface. ICAM- 1 is a cell-adhesion molecule that plays a role in the immunoglobulin migration of endothelial cells in the process of angiogenesis. ICAM- 1 assists in regulating endothelial cell migration during the activation of nitric-oxide synthase, and it regulates the actin cytoskeleton. The relationship between ICAM-1 and the actin cytoskeleton occurs through the interaction of cortactin, $\alpha$-actinin, and ezrin proteins. The actin cytoskeleton could be expected to affect the development of cell polarity and the formation of lamellipodium [10].

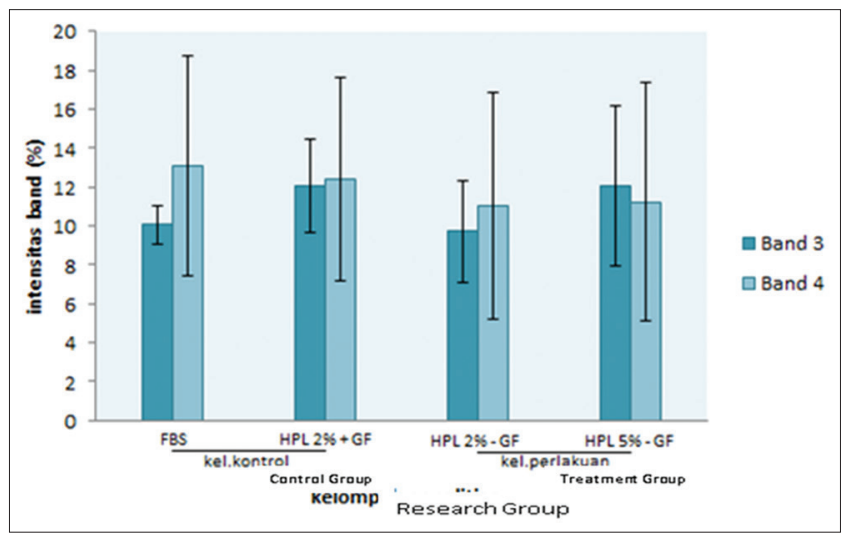

Fig. 1: Average values of intensity for protein bands 3 and 4

Table 1: The average thickness value of protein bands 3 and 4 according to the results of the SDS-PAGE test

\begin{tabular}{llc}
\hline Research group & \multicolumn{2}{l}{ Average Thickness (pixels) } \\
\cline { 2 - 3 } & Band 3 & Band 4 \\
\hline FBS & 11.49 & 37.39 \\
2\%-HPL with a GF & 10.95 & 45.76 \\
2\%-HPL without a GF & 9.32 & 40.90 \\
5\%-HPL without a GF & 11.52 & 52.82 \\
\hline
\end{tabular}

SDS-PAGE: Sodium dodecyl sulfate-polyacrylamide gel electrophoresis,

FBS: Fetal bovine serum, GF: Growth factor, HPL: Human platelet lysate

Table 2: The molecular weight range of proteins bands 3 and 4 according to the results of the SDS-PAGE test

\begin{tabular}{lll}
\hline \multirow{2}{*}{ Research group } & \multicolumn{2}{l}{ Molecular weight range (kDa) } \\
\cline { 2 - 3 } & Band 3 & Band 4 \\
\hline FBS & $78.62-100.88$ & $54.55-73.05$ \\
2\%-HPL with a GF & $89.73-104.64$ & $53.38-76.82$ \\
2\%-HPL without a GF & $90.26-114.22$ & $58.94-83.83$ \\
5\%-HPL without a GF & $88.47-111.56$ & $56.15-80.19$ \\
\hline
\end{tabular}

SDS-PAGE: Sodium dodecyl sulfate-polyacrylamide gel electrophoresis, FBS: Fetal bovine serum, GF: Growth factor, HPL: Human platelet lysate 
VCAM-1 or CD106 is a group of endothelial cell adhesion molecules of immunoglobulin with a molecular weight of $80 \mathrm{kDa}$ [11]. Research conducted on coronary atherosclerotic plaque shows that endothelial cells can express VCAM-1, especially during neovascularization [12]. VCAM1 cells can be induced by interleukin (IL-1), tumor necrosis factor- $\alpha$ (TNF- $\alpha$ ), IL-4, and IL-1. TNF- $\alpha$ plays a role in the migration of endothelial cells and in angiogenesis (in vivo) when implanted in the cornea or chorioallantoic membrane. The mechanism of TNF- $\alpha$ in mediating angiogenesis depends on the secondary mediators, such as prostaglandins, platelet-activating factors, Eck receptor ligand B61, interleukin-8, VEGF, and FGF [13].

CD34 is a transmembrane glycoprotein expressed at the surface of lymphohematopoietic cells as well as stem, progenitor, endothelial, embryonic fibroblast, and other types of cell in fetal and adult neural tissues. The molecular weight ranges from 90 to $120 \mathrm{kDa}$. CD34 expression in cultured HUVEC is allegedly stimulated by VEGF molecules, TNF- $\alpha$, and Notch ligand DLL4. Sialomucin CD34 is expressed and can develop into filopodia, which are characteristic of tip cells. Tip cells trigger blood vessel ends in coordinating multiple processes during angiogenesis [14].

E-selectin or CD62E is a glycosylated transmembrane protein that is expressed by endothelial cells and its molecular weight is $65-85 \mathrm{kDa}$. E-selectin plays a role in the interaction between leukocytes and vascular endothelium in inflammatory conditions, such as rheumatoid arthritis and atherosclerosis. E-selectin also has the ability to interact with endothelial progenitor cells, which have the potential to proliferate and differentiate into mature endothelial cells [15].

Some of the proteins expressed by HUVEC are involved in the angiogenesis process. Angiogenesis plays an important role in bone tissue engineering to form new blood vessels. These blood vessels will serve in the transport of oxygen, nutrients, and hormones, as well as the disposal of waste products [16]. More research is needed to identify the specific proteins using a specific protein marker.

\section{CONCLUSIONS}

From the results of this study, it can concluded that there are no differences among the protein profiles of HUVEC culture media that use FBS and HPL serums with the addition of GFs with HPL and without GFs. Furthermore, 2\%- and 5\%-HPL without a GF can be used as a supplement to the standard HUVEC culture medium.

\section{REFERENCES}

1. Jonsdottir-Buch SM. Platelet lysates manufactured from fresh and expired platelet concentrates as a culture supplement for human. Bone marrow-derived mesenchymal stem cells. Iceland: University of Iceland; 2012.
2. Jonsdottir-Buch SM, Lieder R, Sigurjonsson OE. Platelet lysates produced from expired platelet concentrates support growth and osteogenic differentiation of mesenchymal stem cells. PLoS One 2013;8(7):e68984.

3. Rauch C, Feifel E, Amann EM, Spötl HP, Schennach H, Pfaller W, et al. Alternatives to the use of fetal bovine serum: Human platelet lysates as a serum substitute in cell culture media. ALTEX 2011;28(4):305-16.

4. Bieback K. Platelet lysate as replacement for fetal bovine serum in mesenchymal stromal cell cultures. Transfus Med Hemother 2013;40(5):326-5.

5. Naaijkens BA, Niessen HW, Prins HJ, Krijnen PA, Kokhuis TJ, de Jong $\mathrm{N}$, et al. Human platelet lysate as a fetal bovine serum substitute improves human adipose-derived stromal cell culture for future cardiac repair applications. Cell Tissue Res 2012;348(1):119-30.

6. Chim SM, Qin A, Tickner J, Pavlos N, Davey T, Wang H, et al. EGFL6 promotes endothelial cell migration and angiogenesis through the activation of extracellular signal-regulated kinase. J Biol Chem 2011;286(25):22035-46

7. Park HJ, Zhang Y, Georgescu SP, Johnson KL, Kong D, Galper JB. Human umbilical vein endothelial cells and human dermal microvascular endothelial cells offer new insights into the relationship between lipid metabolism and angiogenesis. Stem Cell Rev 2006;2(2):93-102.

8. Puspitasari AH. Effect of Platelet Shelf-Life on Human Platelet Lysates as FBS Substitute Against Protein Profile of HUVEC Culture Medium. Thesis. Jakarta: Universitas Indonesia; 2013.

9. Eckermann CW, Lehle K, Schmid SA, Wheatley DN, KunzSchughart LA. Characterization and modulation of fibroblast/ endothelial cell co-cultures for the in vitro preformation of three dimensional tubular networks. Cell Biol Int 2011;35(11):1097-10.

10. Kevil CG, Orr AW, Langston W, Mickett K, Murphy-Ullrich J, Patel RP, et al. Intercellular adhesion molecule-1 (ICAM-1) regulates endothelial cell motility through a nitric oxide-dependent pathway. J Biol Chem 2004;279(18):19230-8

11. Cybulsky MI, Iiyama K, Li H, Zhu S, Chen M, Iiyama M, et al. A major role for VCAM-1, but not ICAM-1, in early atherosclerosis. J Clin Invest 2001;107(10):1255-62.

12. Moreno PR, Purushothaman KR, Sirol M, Levy AP, Fuster V. Neovascularization in human atherosclerosis. Circulation 2006;113(18):2245-2.

13. Vanderslice P, Munsch CL, Rachal E, Erichsen D, Sughrue KM, Truong AN, et al. Angiogenesis induced by tumor necrosis factor-AGR; is mediated by alpha4 integrins. Angiogenesis 1998;2(3):265-75.

14. Siemerink MJ, Klaassen I, Noorden CJ, Schlingemann RO. Endothelial tip cells in ocular angiogenesis potential target for anti-angiogenesis therapy. J Histochem Cytochem 2012;61(2):101-5.

15. Oh IY, Yoon CH, Hur J, Kim JH, Kim TY, Lee CS, et al. Involvement of E-selectin in recruitment of endothelial progenitor cells and angiogenesis in ischemic muscle. Blood 2007;110(12):3891-9.

16. Grellier M, Bordenave L, Amédée J. Cell-to-cell communication between osteogenic and endothelial lineages: Implications for tissue engineering. Trends Biotechnol 2009;27(10):562-71. 